\section{PSICOLOGIA IBEROAMERICANA}

\section{Psicología lberoamericana}

ISSN: 1405-0943

psicología.iberoamericana@uia.mx

Universidad Iberoamericana, Ciudad de

México

México

Reyes Ruiz, Norma Elena; Díaz-Loving, Rolando

La virginidad: ¿una decisión individual o un mandato cultural?

Psicología Iberoamericana, vol. 20, núm. 2, julio-diciembre, 2012, pp. 33-40

Universidad Iberoamericana, Ciudad de México

Distrito Federal, México

Disponible en: http://www.redalyc.org/articulo.oa?id=133928816005

Cómo citar el artículo

- Número completo

- Más información del artículo

- Página de la revista en redalyc.org

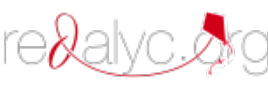

Sistema de Información Científica

Red de Revistas Científicas de América Latina, el Caribe, España y Portugal

Proyecto académico sin fines de lucro, desarrollado bajo la iniciativa de acceso abierto 


\title{
La virginidad: ¿una decisión individual o un mandato cultural?
}

\author{
Virginity: An Individual Decision or a Cultural Mandate?
}

\author{
Norma Elena Reyes Ruiz* \\ Rolando Díaz-Loving** \\ Unidad de InVestigaciones Psicosociales, Facultad de Psicología \\ Universidad Nacional Autónoma de México, México
}

\section{RESUMEN}

La trascendencia y el significado histórico del precepto de la virginidad se extienden a través de todos los grupos culturales. Es así que en la cultura tradicional mexicana se observan las normas y creencias que condicionan y sancionan la práctica sexual con la finalidad de lograr la meta de la virginidad hasta el matrimonio. Por ello, el objetivo del presente estudio fue revisar de qué manera las premisas históricopsicosocioculturales de la virginidad se han ido modificando a lo largo de los últimos 50 años. Para tal efecto se aplicó el instrumento de las Premisas HistóricoSocioculturales (PHSC) (Díaz Guerrero, 2003) a 584 estudiantes de secundaria (266 hombres y 318 mujeres) de varias zonas del Distrito Federal. Finalmente se llevó a cabo una comparación de las mediciones ya hechas en años anteriores (Díaz Guerrero, 2003) con los datos obtenidos del presente estudio. Como segundo propósito, se compararon los puntajes para cada premisa por sexo y por grado escolar.

Descriptores: virginidad, premisas, cultura, creencias, afirmaciones.

\section{ABSTRACT}

The transcendence of virginity has been overwhelming through history. Based on male's need for parental certainty, rituals and traditions evolved to keep virginity norms and believes relevant. In fact, in the Mexican culture -like in many other traditional cultures- there are socio-cultural premises that sanction and reinforce behavioral patterns that insure virginity until marriage. For example, more than 40 years ago, the birth of a baby girl in a Mexican family represented a material and moral loss due to the costs of insuring virginity and a suitable partner. The purpose of this study was to appraise attachment to virginity premises. To achieve the goal, three previous applications of the virginity socio-cultural premises were reviewed (Díaz Guerrero, 2003), and compared with current data from 584 coed high school students in Mexico City. As a second purpose, each premise was compared by sex and school grade.

Keywords: virginity, culture, beliefs, premises, statements.

\footnotetext{
* Para correspondencia: Lic. Norma Elena Reyes Ruiz. Unidad de Investigaciones Psicosociales, Facultad de Psicología, UnAM. Av. Universidad 3004. Col. Copilco-Universidad. C.P. 04510. Delegación Coyoacán. México D.F. Tel. 5622-2326. e-mail: amronelen@hotmail.com

** Dr. Rolando Díaz-Loving. Unidad de Investigaciones Psicosociales, Facultad de Psicología, unam. Av. Universidad 3004. Col. Copilco-Universidad. C.P. 04510. Delegación Coyoacán. México D.F. Tel. 5622-2326. e-mail: rdiazl@unam.mx
} 


\section{INTRODUCCIÓN}

Quizá en la religión católica, el hecho de pregonar, anunciar y establecer el nacimiento de Cristo de una virgen, tuvo la intención de otorgarle trascendencia a la virginidad, ya que desde la antigüedad hubo santas que ofrecieron, como primer testimonio para seguir el camino de la santidad, mantenerse vírgenes (Díaz Guerrero, 2003). El cristianismo elevó el estado virginal a un valor divino, al considerarlo como el grado más sublime de la vida religiosa, y al imponerlo a todos los que se querían consagrar a Dios.

Dados estos antecedentes, en donde se muestra la importancia que la religión otorga al hecho de que una mujer se mantenga virgen, no es de extrañar que en la cultura tradicional mexicana, como indudablemente sucede en muchas otras, existan creencias arraigadas al respecto (Díaz Guerrero, 2003). Al concentrarse en el patrón de hallazgos, una de las primeras cosas que llama la atención, es como dentro de la familia se condicionaban las características y patrones conductuales tradicionales de los hombres y las mujeres (Flores, 2011).

En el caso de las familias mexicanas, tal como refiere Díaz Guerrero (1955), el nacimiento de una niña hace más de 40 años era una tragicomedia, ya que representaba una pérdida material y moral importante; el primer aspecto hacía referencia al hecho de tener que conceder una dote junto con la hija para que ésta pudiera casarse, aunado a que esto agregaba un nuevo integrante (marido). En segundo término está el aspecto moral, que se vincula sólo con la virginidad de la mujer, ya que si ésta tenía relaciones sexuales previas al matrimonio constituía una deshonra familiar y tenía como consecuencia soportar la presencia de una mujer despreciada por la sociedad y por la propia familia, al no ser "casadera" (Rocha, 2008).

Lo indudable es que la virginidad, como premisa de nuestra historia sociocultural, ha estado vigente al menos desde la Colonia (Díaz Guerrero, 2003), si no es que de tiempo atrás. Aun cuando México ha vivido, a partir de los años treinta del siglo XX una tendencia hacia la homogeneización cultural, en virtud de la construcción de la nación a partir de la Independencia, sus características no son uniformes (Amuchástegui, 1998). Ciertos elementos de las antiguas culturas indígenas coexisten con creencias y rituales católicos, y con saberes especializados modernos en una mezcla particular.

El significado y simbolismo del constructo social de la virginidad es innegable tanto para la cosmogonía de las culturas indígenas como para las contemporáneas, aunado también a los significados relacionados con la actividad sexual de parte de las mujeres que no hablan de su propio deseo sexual ni de las sensaciones placenteras, ni de la excitación. Este silencio va a la par de los atributos de pureza y discreción que están muy en armonía con la imagen católica de la madre (Amuchástegui, 1998).

Ya en los estudios de Bataille (1992) sobre el análisis del catolicismo en la cultura occidental, se ofrece una teoría para captar la relación entre la sexualidad y lo sagrado, la cual se resumirá para que se pueda dar espacio al estudio de la cultura mexicana en específico. Para este autor, estudiar el erotismo significa estudiar también la religión, ya que existe una regulación universal de la actividad sexual fincada en las creencias religiosas.

Esta barrera contra el erotismo se apoya en el hecho de que: "lo que está en juego en el erotismo es siempre una disolución de las formas constituidas". Además de considerar los aspectos sexuales como "sagrados" porque ofrecen la posibilidad de la continuidad entre los seres (Marcos, 1989). Se señala entonces que para analizar a la virginidad es importante contemplar tres aspectos importantes: el erotismo, la sexualidad y la religión, factores necesarios para brindar un panorama referencial de lo que sucede con ésta como un constructo sociocultural. Por lo tanto, cabe resaltar que dependiendo del contexto cultural en el que uno se encuentre, será la forma en que se le brinde significado a la virginidad, además de que puede existir un fuerte control sobre ésta en el caso de la mujer (Amuchástegui, 1998).

Dentro de la sociocultura mexicana una serie de mandatos que se han denominado premisas históricosocioculturales son los remanentes de ese control. Todos esos dichos, proverbios, refranes, modos de enfrentar los problemas cotidianos, creencias y prejuicios, los recogió Díaz Guerrero (2003) y los operacionalizó, conformando 123 premisas en donde se identificaron nueve factores: machismo, obediencia afiliativa, virginidad, abnegación, temor a la autoridad, status quo familiar, respeto sobre amor, honor familiar y rigidez 
cultural. Uno de ellos es precisamente el factor de virginidad, que versa sobre el grado de importancia que se le asigna a las relaciones sexuales prematrimoniales; prescribe el valor que para la mujer debe tener mantenerse virgen hasta el matrimonio. La misma premisa ha estado muy estrechamente ligada a creencias tradicionales y religiosas, y en el centro de estas ideas se encuentra la valoración o la estigmatización de la mujer. Por un lado, se ha exaltado la virginidad como un preciado tesoro que determina su pureza. En el polo opuesto, se encuentra el rechazo y el estigma, si llega al matrimonio sin ser virgen (Alarcón, 2005).

Los trabajos realizados con la escala de premisas históricosocioculturales han demostrado que algunas son sensibles a la variable tiempo histórico. Díaz Guerrero $(1974,1995)$ ha comparado resultados obtenidos, en hombres y mujeres, en 1959, 1970 y 1994, en el factor virginidad. El autor encuentra que en la sociedad mexicana ha declinado fuertemente en los últimos 35 años; los datos muestran una tendencia a una mayor libertad de las mujeres en los mandatos tradicionales en comparación con los hombres. También Díaz Guerrero $(1984,1989,1992)$ ha informado que las premisas (HSC) guardan relación con la edad, pues a medida que crece el individuo es menor el efecto de los mandatos sociales; la causa de esto es que la individualidad y la personalidad se hacen más influyentes. Del mismo modo, se han encontrado relaciones estadísticas significativas entre las PHSC y sus escalas factoriales, con variables cognitivas, de personalidad, intereses vocacionales y niveles socioeconómicos. Se encontró, por ejemplo, en la Ciudad de México y en provincia, que los factores de las PHSC's correlacionaban significativamente alto con la edad en pruebas de inteligencia, como el WISC y las matrices progresivas de Raven. Correlaciones similares se obtuvieron entre factores de las PHSC's y la prueba de manchas de tinta de Holtzman, el test de ansiedad y el inventario de personalidad de Jackson (Díaz Guerrero, 2003).

En otro estudio llevado a cabo por Alarcón (2005) se aplicó una versión adaptada de la escala de PHSC's de la familia mexicana a 410 estudiantes peruanos, varones y mujeres, de 20 a 30 años. Dicho autor encontró que en un estrato socioeconómico alto se manifiesta un claro rechazo de las pautas tradicionales de virgini dad y en cambio, hay mayor conservadurismo entre varones y mujeres de nivel socioeconómico bajo, considerando el nivel educativo como un aspecto más en el nivel socioeconómico. Además de señalar que las premisas históricosocioculturales que se compararon en dos culturas, Perú y México, presentan cambios en las creencias culturales acerca de la virginidad. Ésta ha estado muy estrechamente ligada a creencias tradicionales y religiosas, encontrándose en el centro de estas ideas la valoración o la estigmatización de la mujer. Por un lado, se ha exaltado la virginidad como un preciado tesoro que determina su pureza. En el polo opuesto, se encuentra el rechazo y el estigma si llega al matrimonio sin ser virgen.

El presente trabajo tiene como objetivos: 1) comparar los porcentajes de la muestra actual de estudiantes de secundaria con las mediciones de las tres aplicaciones anteriores (1959, 1970 y 1994) llevadas a cabo por Díaz Guerrero (2003); y 2) estudiar el factor de virginidad por sexo y grado escolar en estudiantes de secundaria del Distrito Federal.

\section{MÉTODO}

\section{Participantes}

La escala de Premisas HistóricoSocioCulturales fue administrada a una muestra intencionada de 584 estudiantes de secundaria, 266 hombres y 318 mujeres de secundarias mixtas y públicas de varias zonas del Distrito Federal, cuyas edades variaban entre los 15 a 17 años.

\section{Instrumento}

Se aplicó el Inventario de Premisas HistóricoSocioCulturales, compuesto por 123 reactivos, que fue sometido a análisis factorial, con lo que se logró identificar nueve factores básicos; sin embargo sólo se trabajó con el factor de virginidad por motivos del presente estudio. Consta de una forma de respuesta dicotómica (ausencia-presencia); se muestra a los participantes una serie de oraciones y tienen que marcar sólo aquellas que coincidan con su sistema de creencias (Díaz Guerrero, 1986).

\section{Procedimiento}

Se solicitó un permiso previo a las autoridades de las escuelas, programando los horarios en los que se asis- 
tiría al lugar. Una vez que se llevaron a cabo dichos procedimientos, se aplicó la prueba en los grupos programados; la aplicación fue grupal. A cada alumno se le entregó un cuestionario y un lápiz; el facilitador les leía las instrucciones de la escala, así como la forma de responder, además de resolver dudas en caso de que existieran. Se les pidió marcar con una $\mathrm{X}$ sólo aquellas afirmaciones con las que estaban de acuerdo, y específicamente, se les dijo: "Si no estás de acuerdo con la afirmación, no la marques".

La aplicación se realizó en los tres grados escolares $\left(1^{\circ}, 2^{\circ}\right.$ y $\left.3^{\circ}\right)$, en secundarias públicas de la Ciudad de México cuidadosamente escogidas para representar tanto rumbos de la ciudad como características económicas de la población. Las secundarias fueron mixtas, y se prefirió que fueran públicas, porque eran más representativas de la población mexicana que las privadas. Si los estudiantes no estaban de acuerdo en contestar el instrumento o por alguna razón no querían continuar con el mismo, podían salir en cualquier momento.

\section{RESULTADOS}

\section{Porcentajes de las premisas del factor virginidad}

Se realizó un análisis de frecuencias de las premisas del factor virginidad. Para efectos de presentar las comparaciones con las mediciones de los años anteriores (Díaz Guerrero, 2003) se muestran los datos en porcentajes en las gráficas de la 1 a la 6 .

Gráfica 1. "Una mujer debe ser virgen hasta que se case"

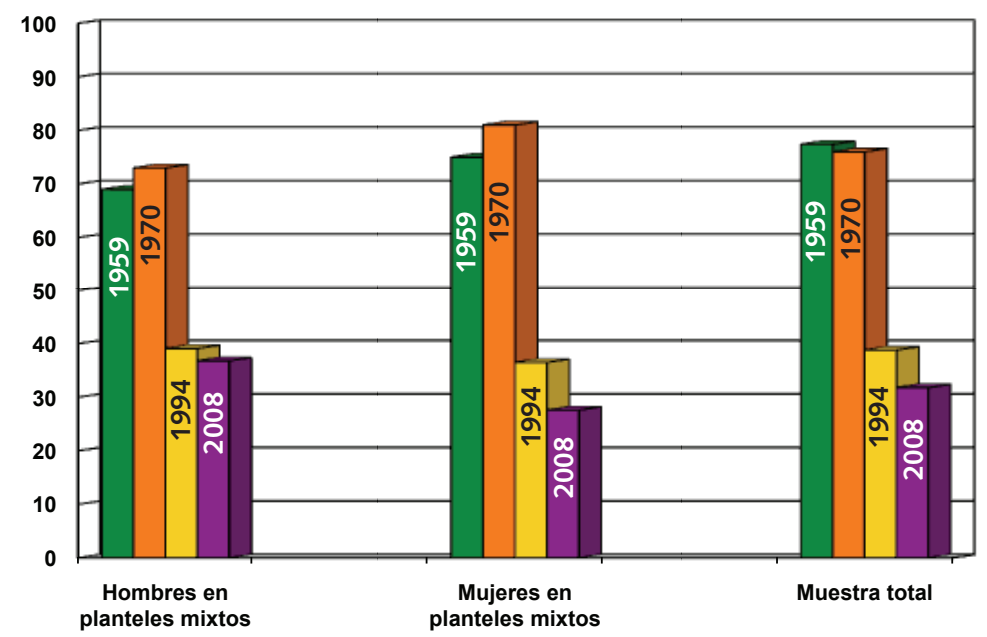

Gráfica 2. "La mayoría de los hombres no se casan si la mujer no es virgen"

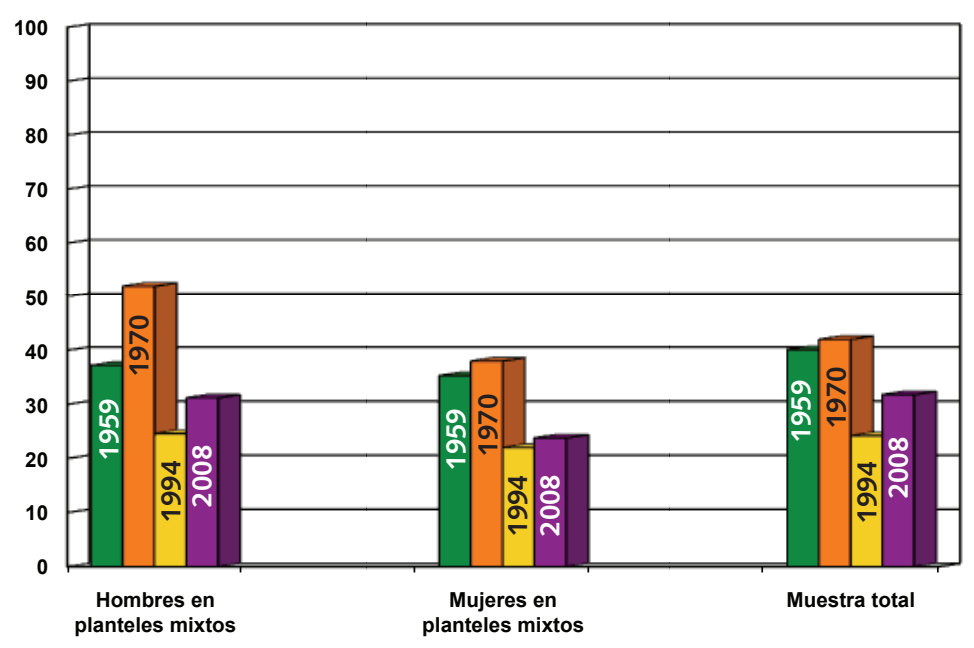


Gráfica 3. "A todo hombre le gustaría casarse con una mujer virgen"

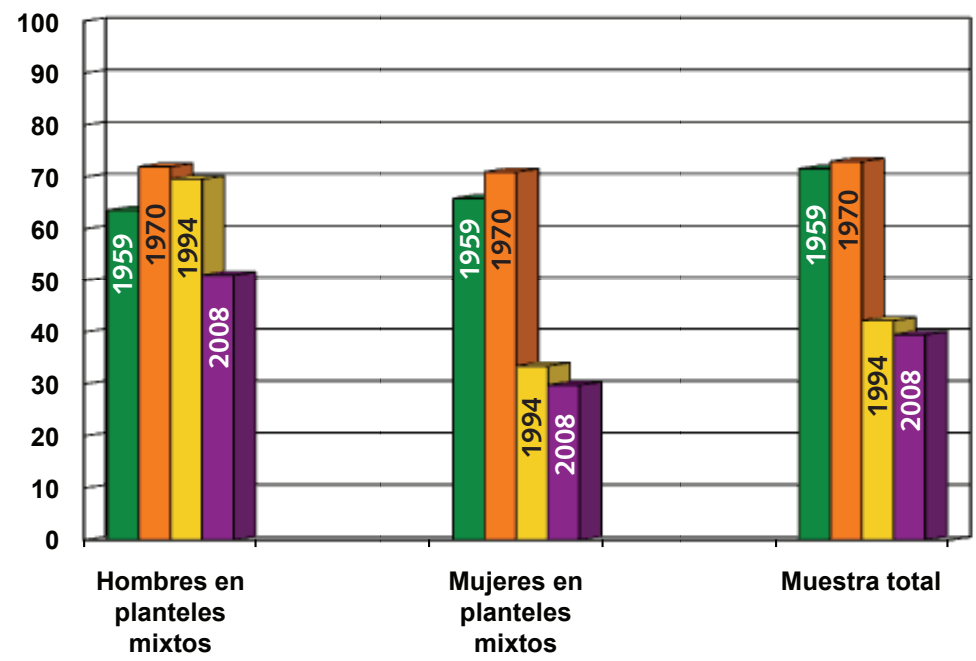

Gráfica 4. "Todas las mujeres deben permanecer vírgenes hasta el matrimonio"

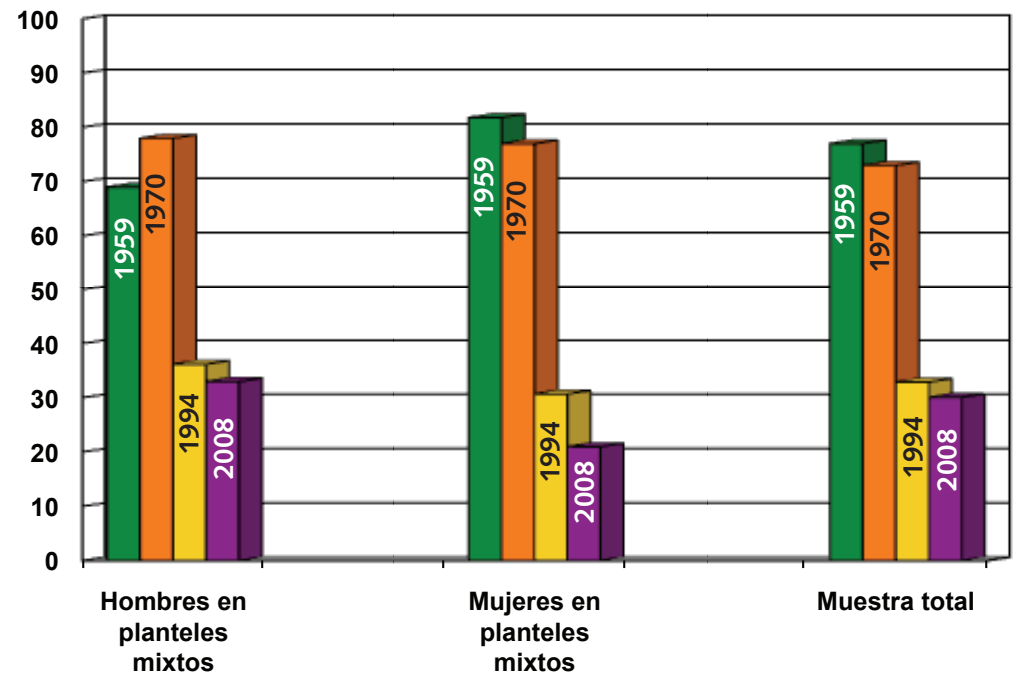

Gráfica 5. "Ser virgen es de gran importancia para la mujer soltera"

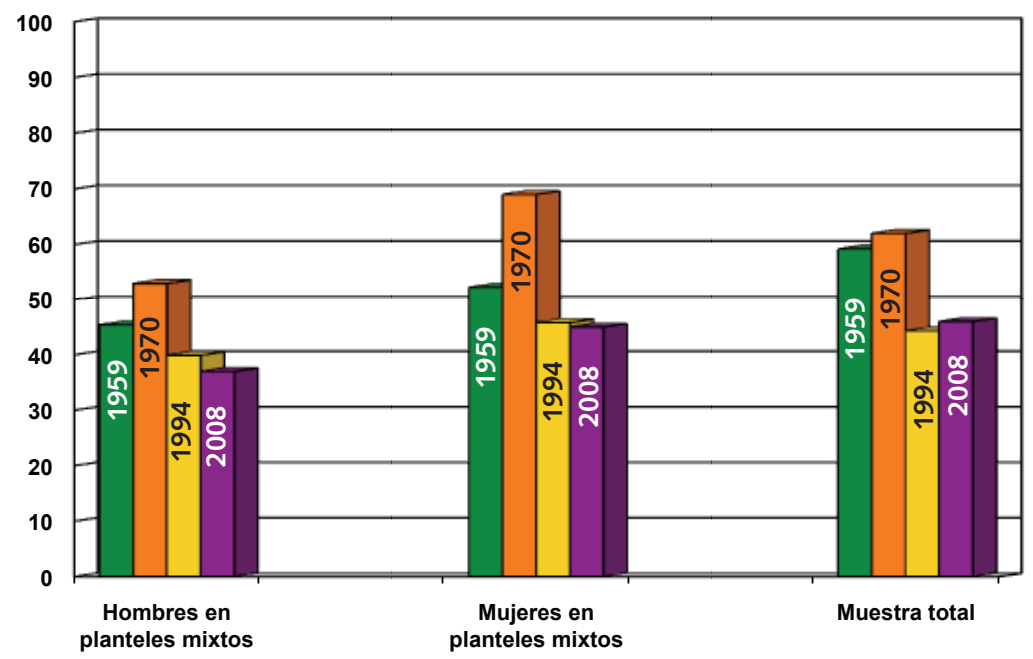


Gráfica 6. “Una mujer soltera que ha perdido su virginidad no será una esposa tan buena como una mujer soltera que es virgen"

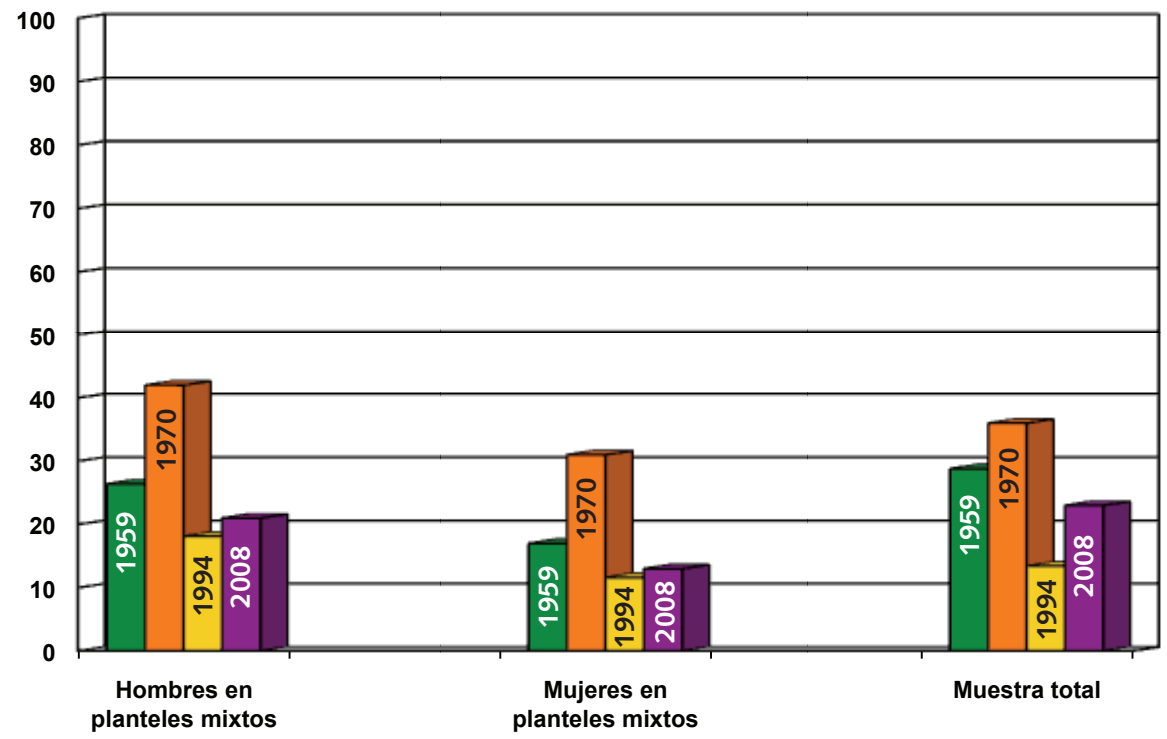

Análisis de diferencias por sexo y escolaridad en el factor virginidad para la muestra del 2008

Con la finalidad de observar el nivel de apego al factor virginidad (la suma de las seis premisas) en hombres y mujeres de los tres grados escolares de secundaria se llevó a cabo un análisis de varianza de 2 (sexo) por 3 (grado escolar). En la tabla 7 se muestran los valores de la media para hombres y mujeres en cada grado escolar, así como la media total para cada uno de los grupos. Se encontraron efectos significativos para sexo $(\mathrm{F}=10.19 ; \mathrm{p} .001)$ y para escolaridad $(\mathrm{F}=6.84 ; \mathrm{p} .001)$.

\section{DISCUSIÓN}

Haciendo referencia a los objetivos de este estudio, y en relación únicamente con los obtenidos para dicha muestra, se puede señalar lo siguiente: en primer lugar, de acuerdo con los datos de las gráficas donde se muestran las comparaciones de las tres etapas anteriores de la aplicación de las PHSC contrastándolas con datos de este nuevo siglo, para el factor virginidad: una mujer es virgen cuando llega al matrimonio sin haber tenido ninguna relación sexual. A diferencia de lo que ocurría en años anteriores (1959, 1970 y 1994), en la aplicación actual existe una tendencia a la disminución de las premisas: "una mujer debe ser virgen hasta que se case" y "todas las mujeres deben permanecer vírgenes hasta el matrimonio" en chicos de secundaria, además de que las mujeres, a diferencia de los varones, ya no están tan de acuerdo en seguir dichas premisas. Es interesante observar que, después de 14 años (considerando la aplicación de 1994), en el caso de la premisa: "la mayoría de los hombres no se casan si la mujer no es virgen" tuvo un incremento para la aplicación actual,

Tabla 1. Factor virginidad.

\begin{tabular}{|c|c|c|c|}
\hline SECUNDARIA & HOMBRES & MUJERES & MEDIA TOTAL \\
\hline $1^{\circ}$ & 1.40 & 1.31 & $\mathrm{X}=1.35$ \\
\hline $2^{\circ}$ & 1.34 & 1.27 & $\mathrm{X}=1.30$ \\
\hline $3^{\circ}$ & 1.27 & 1.20 & $\mathrm{X}=1.23$ \\
\hline MEDIA TOTAL & $\mathrm{X}=1.35$ & $\mathrm{X}=1.26$ & \\
\hline
\end{tabular}


y los hombres la aceptaron en mayor medida que las mujeres. Consideraron de vital importancia que la mujer con la que se vayan a casar sea virgen; sin embargo cabe resaltar que los porcentajes estaban por debajo del 50\% para dicha muestra. En el caso de la premisa: "a todo hombre le gustaría casarse con una mujer virgen", el $50 \%$ de los hombres de la muestra actual dicen estar de acuerdo con dicho oración, a diferencia de un 30\% por parte de las mujeres. En comparación con las mediciones anteriores $(1959,1970$ y 1994) existe un decremento, sin embargo esta es una de las premisas que puntúa con un porcentaje más elevado. Con respecto a la afirmación "ser virgen es de gran importancia para la mujer soltera", las chicas de secundaria en las cuatro evaluaciones (1959, 1970, 1994 y 2008) presentan un elevado porcentaje, a diferencia de los varones. Por último, e la premisa: "una mujer soltera que ha perdido su virginidad no será una esposa tan buena como una mujer soltera que es virgen" es la única que presenta menores porcentajes comparada con el resto de las afirmaciones que conforman el factor de virginidad, además de que las mujeres le dan menor importancia que los varones de secundaria.

Analizando dichas premisas se puede expresar que con el paso del tiempo las afirmaciones de virginidad han sido permeadas por los cambios sociales, el desarrollo tecnológico y científico, así como la cada vez más frecuente inserción de la mujer en el ámbito laboral, ya que hay una mayor tendencia al desapego de las premisas en las mujeres en comparación con lo que se observa en los datos de los hombres. Además de señalar que ha disminuido la aceptación de las premisas de virginidad tanto para hombres y mujeres en estos últimos 40 años, no hay que perder de vista también que se trata de estudiantes de secundaria y que ellos son la fuente de vanguardia y empuje para poder ir observando cómo es que llegan con un bagaje de significados, creencias y valores (premisas HSC), y como estos se modifican conforme pasan de un grado escolar a otro. Por ello, para el segundo objetivo se corrió un análisis de varianza (ANOVA) para hombres y mujeres de primero, segundo y tercero de secundaria, y se observó que de acuerdo a las medias, los chicos que están en primer grado, y más hombres que mujeres, presentan un mayor apego a las premisas del factor de virginidad, y conforme van pasando de grado escolar es menor la adherencia a dichos mandatos y sobre todo en las mujeres.

En definitiva, la decisión contracultural de los individuos le ha ganado terreno, a través de los años, a los mandatos socioculturales, lo que deja ver una tendencia progresiva a que el control externo de la sociocultura quede en manos del control interno, o la también llamada motivación intrínseca de los individuos (Alarcón, 2010). Además de agregar que en lo concerniente a los efectos diferenciales de la socialización en hombres y mujeres, así como el impacto del proceso educativo formal, se hace patente que las transformaciones han llegado a las mujeres antes que a los hombres, y que como ya lo señalaba Díaz Guerrero (2003), la educación laica aleja a las personas de las premisas tradicionales. En este sentido, a mayor educación y el ser mujer implica, entre otras cosas, una visión más autoafirmativa, mayor desarrollo de la autonomía, una perspectiva más equitativa, un menor apego a las premisas cardinales del pasado: supremacía del padre, abnegación de la madre y obediencia afiliativa de los hijos (Díaz-Loving, Rivera Aragón, Villanueva \& Cruz, 2011). 


\section{REFERENCIAS}

Alarcón, R. (2005). Premisas históricosocioculturales de la juventud peruana: Obediencia filial y virginidad. Revista Latinoamericana de Psicología, 16(1), 81-94.

Amuchástegui, A. (1998). La dimensión moral de la sexualidad y de la virginidad en las culturas híbridas mexicanas. Relaciones, 19, 102-133.

Bataille, G. (1992). El erotismo. México: Tusquets.

Díaz-Guerrero, R. (1955). Neurosis of mexican family structure american. Journal of Psychiatry, 112, 411417.

Díaz-Guerrero, R. (1959). Mexican assumptions about interpersonal relations. A Review of General Semantics, $112(2)$.

Díaz-Guerrero, R. (1974). La mujer y las premisas históricosocioculturales de la familia mexicana. Revista Latinoamericana de Psicología, 6, 7-16.

Díaz-Guerrero, R. (1984). La psicología de los mexicanos. Un paradigma. Revista Mexicana de Psicología, 1(2), 95-104.

Díaz-Guerrero, R. (1986). El ecosistema sociocultural y la calidad de vida. México: Trillas.

Díaz-Guerrero, R. (1989). Una etnopsicología mexicana. Ciencia y desarrollo, 15, 69-85.

Díaz-Guerrero, R. (1992). El desarrollo de la personalidad en México. Implicaciones para las teorías de la personalidad. Conferencia magistral invitada. Congreso
Iberoamericano de Psicología. Madrid, España, Julio, 5-10.

Díaz-Guerrero, R. (1995). Una aproximación científica a la etnopsicología. Revista Interamericana de Psicología, 27, 359-389.

Díaz-Guerrero, R. (2000). La evolución del precepto de la virginidad. Este País, 30-33.

Díaz-Guerrero, R. (2003). Bajo las garras de la cultura. Psicología del mexicano 2. México: Trillas.

Díaz-Guerrero, R. (2003). Psicología del mexicano. Descubrimiento de la etnopsicología. México: Trillas.

Díaz-Loving, R., Rivera Aragón, S., Villanueva, G.B.T \& Cruz, L.M. (2011). Las premisas histórico-socioculturales de la familia mexicana: su exploración desde las creencias y las normas. Revista Mexicana de Investigación en Psicología. 3(2), 128-142.

Flores, M.M. (2011). La cultura y las premisas de la familia mexicana. Revista Mexicana de Investigación en Psicología. 3(2), 148-153.

Marcos, S. (1989). Curas, dioses y erotismo: el catolicismo frente a los indios. En A. M. Portugal. Mujeres a iglesia. Sexualidad y aborto en América Latina. México: Fontamara.

Rocha, T.E. (2008). Cultura de género y sexismo: de Díaz Guerrero al posmodernismo. En R. Díaz-Loving. Etnopsicología mexicana: siguiendo la huella teórica y empírica de Díaz Guerrero. México, DF: Trillas. 